http://jmscr.igmpublication.org/home/ ISSN (e)-2347-176x ISSN (p) 2455-0450

crossref DOI: https://dx.doi.org/10.18535/jmscr/v8i4.11

\title{
Role of Prophylactic Use of Single Dose Antibiotic in Preventing Surgical Site Infection in Elective Surgery
}

\author{
Authors \\ Dr Ravi Ranjan", Dr Amit Gupta ${ }^{2}$, Dr Md Wahhaj ${ }^{3}$, Dr Nirbhay Singh ${ }^{4}$ \\ ${ }^{1}$ Assistant Professor Dr RML Hospital New Delhi \\ ${ }^{2}$ Consultant Critical Care Medicine Max Superspeciality Hospital Shalimarbagh New Delhi \\ ${ }^{3}$ Senior Resident DMCH \\ ${ }^{4}$ Post Graduate Surgery Resident \\ Corresponding Author \\ Dr Md Whhaj
}

\begin{abstract}
Background: Surgical site infections (SSIs) are a common cause of health-related infection and are associated with extended hospital stays and increased costs. The incidence of surgical site infections is affected by factors such as bacterial inoculation and virulence, host defenses, preoperative treatment and intraoperative management. Antimicrobial prophylaxis has been recognized as a standard procedure to eliminate postoperative risks of clinical practice due to infections. Our research was designed to determine the impact of prophylactic antibiotics on patient's elective postoperative wound and to analyze the result.

Methods: A prospective study that includes 200 patients admitted to the Department of Surgery at Dr RML Hospital New Delhi from March to August 2019 undergoing elective surgery was conducted. The research involved patients who underwent elective surgery. Preoperatively, ceftriaxone was used 30 minutes before incision and its impact on postoperative wound infection was studied.

Results: In our sample, we had 200 cases mainly males of 20-40 years of maximum age range. After administering antimicrobial prophylaxis, 07\% of the maximum 200 patients experienced SSI.

Conclusions: A single dose of Ceftriaxone prophylaxis antibiotics is effective in preventing uncomplicated surgical site infection in an elective situation assuming an uncomplicated procedure.
\end{abstract}

\section{Introduction}

SSIs are the most frequent infections associated with health care and are the costliest. SSIs are responsible for 38 percent of nosocomial infections in surgical patients. Wound infection is one of the most common complications of surgery and causes up to $5 \%$ of all surgical procedures. They are referred to as surgical site infection (SSI) and account for a quarter of all nosocomial infections. In terms of hospital stay, pain, reintervention, and cost, SSI adds to the morbidity. ${ }^{[1]} \mathrm{A}$ variety of risk factors that contribute to SSI-intrinsic (patient-related) and extrinsic (procedure-related) have been established. Many of the patient-related factors are non-modifiable, such as age, co-morbidities (diabetes, chronic re). Since the surgical site is a possible port for exogenous microbes, this presents an immediate threat to the skin and complications which contribute to delayed healing of wounds. (Mangram et al., 1999; Berrios-Torres et al., 2017) ${ }^{3}$. The preoperative stage is known to 
be the most crucial period of a procedure in which the aim is to reduce the bacterial load surrounding the area of incision. The use of antibiotics before surgery is considered effective in the prevention of SSIs, which are among the most common complications preventable after surgery. ${ }^{4} \mathrm{By}$ demonstrating antibiosis, Joseph Lister greatly reduced the rate of disease and presented surgeons with a tool to fight infections. The invention of antibiotics further revolutionized the surgery field. Yet through understanding of infection control and improvements in clinical procedure, postoperative infection remains a problem that every surgeon faces. A patient with SSIs has a risk of being admitted to intensive care by more than 60 percent and is 15 times more likely to be readmitted to the hospital within 30 days after discharge. The use of antimicrobial prophylaxis (AMP) has been long advocated as a means of preventing post-operative infections. The rationale behind the use of peri-operative antimicrobial prophylaxis is that if present in sufficient concentration in the tissue, antibiotics boost the immune system and help eliminate any bacteria that may be present in these surgical sites. Serum concentrations of some antimicrobials tend to drop rapidly during certain surgical procedures while the concentration of others hardly get affected.Throughout cardiothoracic surgery, the use of cardiopulmonary bypass significantly lowers the plasma vancomycin level and thus increases the risk of acquiring SSIs in these patients. Specific recommendations have been developed for the proper use of AMP, covering a wide range of surgical procedures. ${ }^{6-9}$ Most of these recommendations advise the use of cephalosporins, particularly those with anaerobic activity as the drug of choice, but antibiotic preference may vary depending on the bacterial flora at the incision site, antibiotic penetration at the site, and allergic profile of the person.As suboptimal levels of antibiotics in the tissue significantly increase the risk of SSI, the correct timing of antimicrobial administration prior to surgical incision is crucial. The exact timing of the administration of antibiotics varies from 30 minutes to 2 hours before the incision.According to the Surgical Care Improvement Project (SCIP), for most antimicrobials except for flouroquinolones and vancomycin, the ideal time for antibiotic administration should be 1 hour before incision, for which 2 hours is considered the ideal time.

\section{Methods}

The present study was conducted from March 2019 to August 2019 at Dr. RML Hospital New Delhi after obtaining approval from the Ethical Committee of the Institute. Included in the study were 200 consecutive surgical patients undergoing clean or clean contaminated surgery. All participating patients gave informed written consent and those who were not willing to consent were not included. Those not included in the analysis were patients under 18 years of age, patients with unexplained co-morbidity and patients with allergies. In order to rule out any indication or effect of disease, patients were examined thoroughly. Proper history of treatment was taken from patients to ensure that 14 days before the surgical procedure, none had received any antibiotics.1.0 gm intravenous ceftriaxone antimicrobial prophylaxis was administered 30 minutes prior to incision. There were no doses of antimicrobials given after surgery. Surgery was performed with proper asepsis and at the end of the surgery, surgical wound dressing was performed with proper sterile dressing.Postoperative day 2 surgical wound dressing was done using stringent aseptic technique. The surgical area was subsequently left open for review. All patients are checked for any signs or symptoms of surgical site infection up to 30 days after surgery. The person was said to have SSI when he fulfilled the criteria of SSI as defined by the Center for Disease Control and Prevention. ${ }^{12}$ When medical signs and symptoms of illness are present, the specimen was obtained using two sterile swab sticks after thoroughly cleaning the injury. Samples were sent for identification and 
antimicrobial susceptibility report to the microbiology laboratory. Antibiotics were prescribed for all patients developing SSI to visit the hospital for regular wound dressing and oral antibiotics. By dividing the total number of SSIs by the total number of surgeries, the SSI rate was calculated.

\section{Result}

During the period between March 2019 to August 2019, 200 consecutive elective surgical cases were included in the study. Out of the total 200 patients 14 (7\%) developed SSI despite antimicrobial prophylaxis. There was female preponderance in the study with 110 out of total 200 included patients being females. The mean age of the patients was 37 years. Most of the patients undergoing surgery were in the age group $20-40$ years. Highest incidence of infections was recorded in age between 51- 60 years.

Fig 1 Relation of SSI with Age

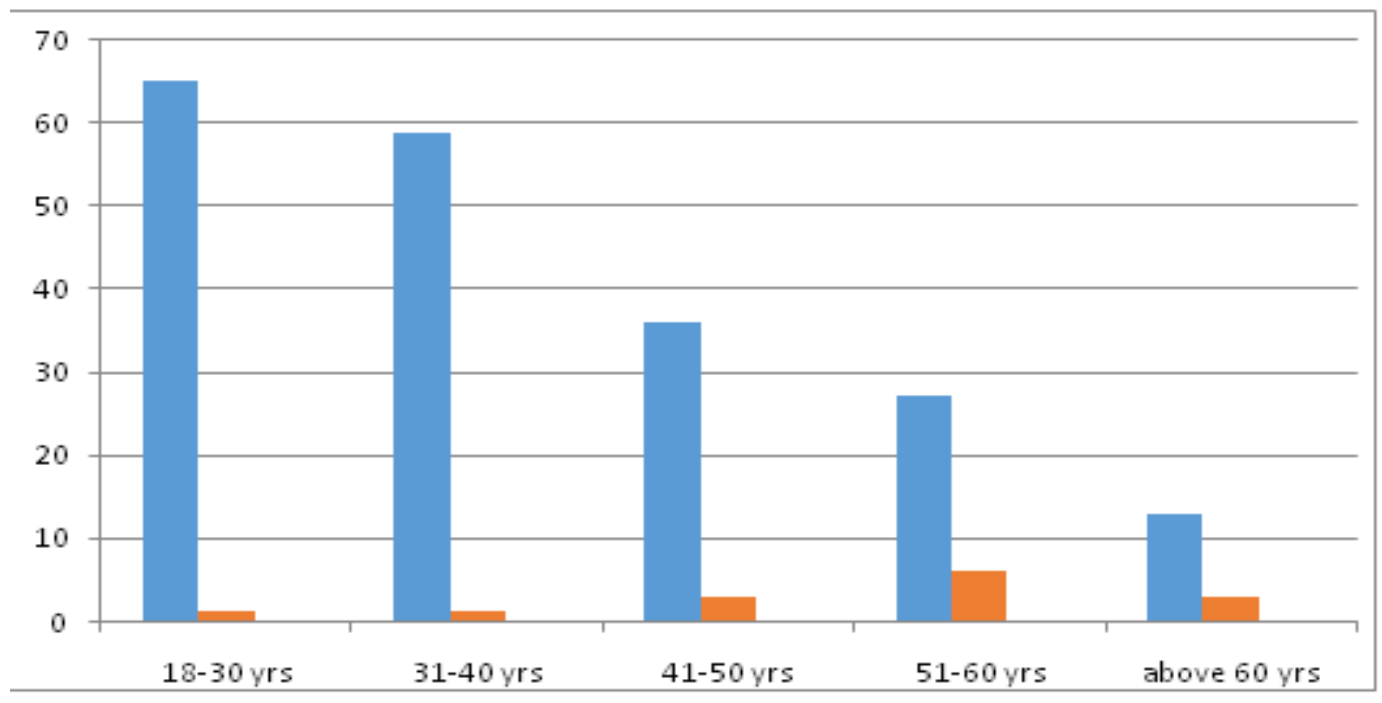

No of Patients with different age group

0 Incidence of SSI among various age groups

A total of 78 clean and 122 clean contaminated surgeries were performed during the study period. The most common surgery performed in clean surgeries was hernioplasty (29). Among the clean contaminated surgery cholecystectomy was the most common surgery performed.

Total Numbers Of Surgeries Performed

\begin{tabular}{lcc|}
\hline Name Of Surgery & \multicolumn{2}{c|}{ Surgery Performed } \\
Cholecystectomy( Lap) & Total Number & Percentage \\
Chokecystectomy (Open) & 61 & $30.50 \%$ \\
Appendicectomy (Lap) & 21 & $10.50 \%$ \\
Appendicectomy(Open) & 06 & $03.00 \%$ \\
Cystolithotomy & 26 & $13.00 \%$ \\
Lipoma excision & 05 & 02.50 \\
Hernioplasty Lichtenstein & 02 & $01 \%$ \\
TAPP (Lap Hernia) & 36 & $18 \%$ \\
Ventral Hernia Mesh Repair & 10 & $05 \%$ \\
Umbilical Hernia Mesh Repair & 15 & $07.50 \%$ \\
Sebaceous Cyst Excisions & 11 & $05.50 \%$ \\
Hydrocele & 02 & $01.00 \%$ \\
Fibroadenoma Excision & 03 & $01.50 \%$ \\
\hline
\end{tabular}




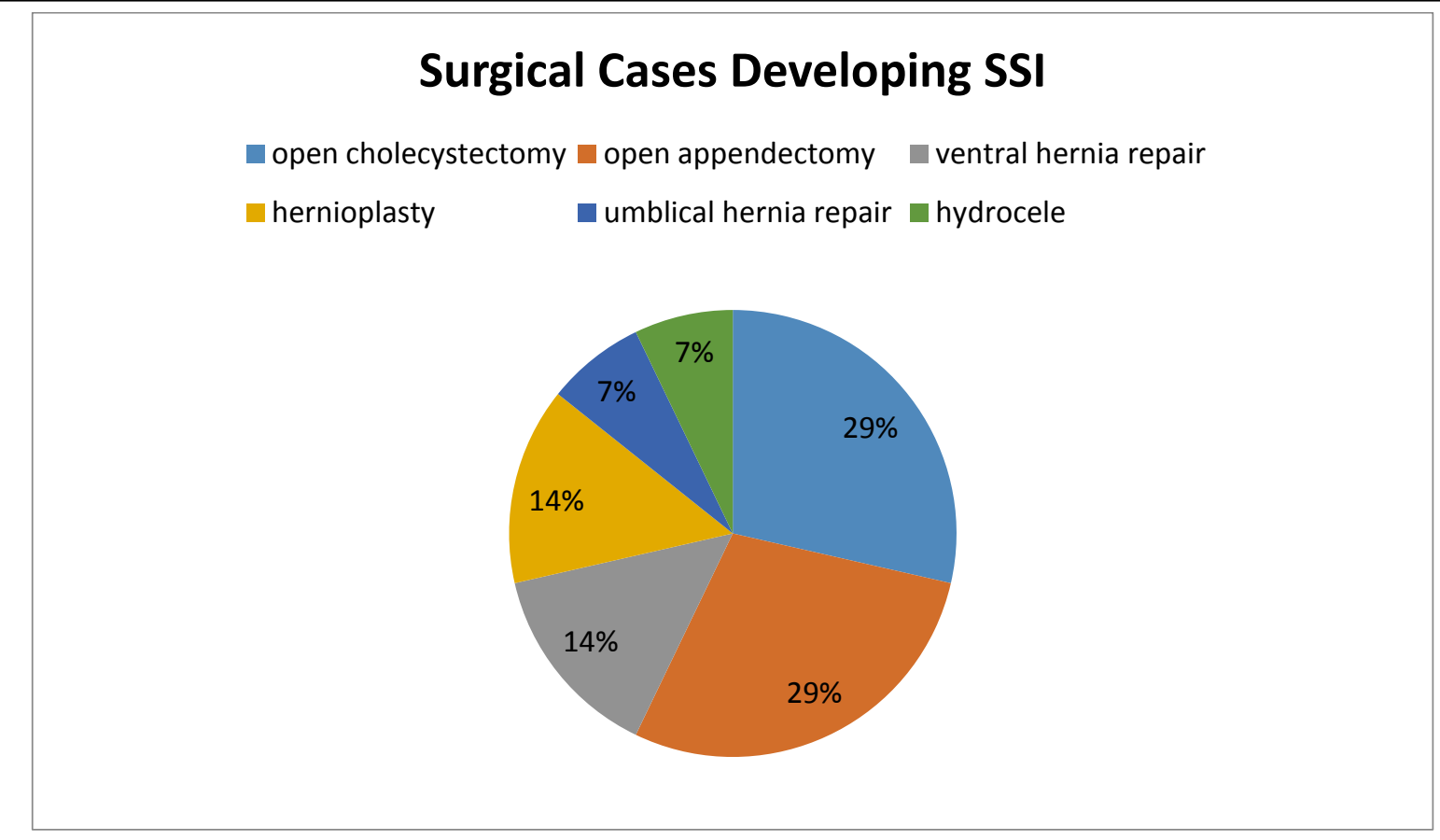

Fig 2 Surgical cases developing SSI

Surgical cases developing SSI - four cases each of open cholecystectomy and open appendectomy two cases each of hernioplasty and ventral hernia repair and one each case of umblical hernia repair and hydrocele developed SSI.

\section{Bacteriology of SSI}

\begin{tabular}{|l|l|}
\hline \multicolumn{1}{|c|}{ Name of surgery } & Organisms isolated \\
\hline Cholecystectomy & E.Coli \\
Appendectomy & Pseudomonas aeruginosa \\
Ventral hernia mesh repair & Staphylococcus aureus \\
Hernioplasty & Staphylococcus aureus \\
Umblical hernia mesh repair & Staphylococcus aureus \\
Hydrocele & Acinobacterbaumanni \\
\hline
\end{tabular}

The most common bacteria isolated from the samples was Staphylococcus aureus which was followed by aeruginosa. S. aureus was isolated from case of hernioplasty, umblicalhenia mesh repair and ventral hernia mesh repair.

\section{Discussion}

Surgical site infections are an important type of infection associated with health care and their monitoring is part of infection control measures in most hospitals. SSI rates are commonly used in hospitals to evaluate the quality of assessment for infection control. ${ }^{13}$ The rate of infection ranged from 2.5 percent to 26 percent. ${ }^{14-18}$ In the present study, the infection rate was $07 \%$. This wide variation in infection rates can refer to the disparity in wound contamination form and how successful post-discharge surveillance (PDS) is being applied. In an analysis of 62,939 procedures, Foord noticed that the average incidence rate of surgical wounds was $4.7 \%$ and the incidence rate was $1.5 \%, 7.7 \%, 15.2 \%$ and $40 \%$ in clean, clean contaminated, contaminated and dirty operations. The study clearly shows that the incidence of SSI in dirty operating procedures was highest. Thus the incidence of SSI would be higher in those centers where more cases of dirty operational procedures were performed compared to those centers where more number of clean performed. In determining the SSI rate, post discharge surveillance is an important parameter. Scottish Surveillance of Healthcare Associated 
Infection Programme (SSHAIP) conducted a study between April 1, 2002, and June 30, 2004 to demonstrate the importance of PDS. ${ }^{20}$ Of the 8,825 non-PDS operations, the SSI value was 2.61 percent, which was significantly lower than the SSI rate reported among the 12,885 PDS operations (6.34 million). Some reports recommended using a single dose of antimicrobial prophylaxis with a broad antibiotic range to stop SSI from developing. The suggestion is that misuse of antibiotics is associated with adverse effects and resistance to bacteria in addition to increasing the patients ' economic burden. The patients who had Clostridium difficile had received antimicrobial prophylaxis and 58\% among these were administered tIn instrumented sepinal fusion surgeries, Marimuthu et al. ${ }^{[1]}$ recommended 24-h antimicrobial prophylaxis was as effective as 72-h. In another analysis by Kim et al. ${ }^{[14]}$ the efficacy of 48-h antimicrobial therapy was contrasted with the efficacy of 72-h treatment and it was suggested that antimicrobial prophylaxis be as effective for $48 \mathrm{~h}$ as $72 \mathrm{~h}$. Musmar et al. suggested that antibiotic use should be stopped within 24 hours of the end of the surgery in order to arrest the onset of resistance against antibiotics, thus exhibiting the adverse effect of antibiotic misuse. After reducing AMP from 24 hours to single dose, Silva Nunes ${ }^{14}$ and colleagues examined the effect on hospital costs. They found that reducing the dose for antimicrobial prophylaxis contributes to monthly hospital cost savings of $\$ 2,000$. Such statistics are very relevant in resource-limited countries such as ours because pursuing single-dose AMP may decrease the impact without increasing the risk of SSI.

\section{Conclusion}

Our study indicates the necessity of antibiotics prophylaxis so as to prevent surgical site infections in cases of elective surgery. Since there is a lack of properly ventilated and cleaned operation theatres in India, it is of at most importance to conducts a number of studies in different parts of the country to collectively access the duration for antibiotics prophylaxis.

\section{Financial support and sponsorship: Nil.}

Conflicts of interest: There are no conflicts of interest.

\section{Reference}

1. Zumla A. Mandell, Douglas, and Bennett's principles and practice of infectiou Diseases. 2010;10(5):303

2. Kirkland KB, Briggs JP, Trivette SL, of surgicalsite infections in the 1990s: Attributable mortality, excess length of hospitalization, and extra costs. Infect Control Hosp Epidem

3. Berríos-Torres SI, Umscheid CA, Bratzler DW, et al. Centers for Disease Control and Prevention Guideline for the Prevention of Surgical Site Infection, 2017. JAMA Surg 2017; $152: 784$

4. Global guidelines for the prevention of surgical site infection.World Health Organization 2016 https://www.ncbi.nlm.nih.gov/pubmedheal th/PMH0095752/pdf/PubMedHealth_PM H0095752.pdf (Accessed on September 19, 2017).

5. Miles AA, Miles EM, Burke J. The value and duration of defence reactions of the skin to the primary lodgement of bacteria. Br J Exp Pathol

6. Burke JF. The effective period of preventive antibiotic action in experimental in Surgery.1961; 50: 161168.

7. Ortega GM, Marti-Bonmati E, Guevara SJ, Alteration of vancomycin pharmacokinetics during cardiopulmonary bypass in patients undergoing cardiac surgery. Am J Health Syst Pharm

8. Bratzler DW, Houck PM. Antimicrobial prophylaxis for surgery: An advisory statement from the National Surgical 
Infection Prevention Project. 2004; 38:1706-1715.

9. Gilbert DN, Moellering RC Jr, Eliopoulos GM, The Sanford Guide to Anti Sperryville, VA: Antimicrobial Therapy Inc.; 2008.

10. Antimicrobial prophylaxis in surgery [SIGN publication No. 45]. Scottish Intercollegiate Guideline Network Website.

http://www.sign.ac.uk/guidelines/fulltext/4 5/index.html. Accessed Au

11. Polk HC Jr, Christmas B. Prophylactic antibiotics in surgery and surgical wound infections.66:105-111.

12. Bratzler DW, Hunt DR. The surgical infection prevention and surgical care improvement projects: National initiatives to im having surgery. Clin Infect Dis

13. Steinberg JP, Braun BI, Hellinger WC, antimicrobial prophylaxis and the risk of surgical site infections: Results from the Trial to Reduce Antimicrobial Prophylaxis Errors (TRAPE). 2009.

14. Olson MM, Lee JT Jr. Continuous, 10-year wound infection surveillance. Results, advantages, and unanswered questions. Arch Surg 1990; 125:794-803.

15. Silvia Nunes Szente Fonseca, So^nia Regina Melon Kunzle, Maria Jose' Junqueira, Renata Teodoro Nascimento, Jose' Ivan de Andrade, Anna S. Levin.Implementing 1-Dose Antibiotic Prophylaxis for Prevention of Surgical Site Infection.Arch Surg. 2006;141:1109-1113. 